\title{
A disturbance attenuation approach for the control of differential-algebraic systems
}

\author{
Pierluigi Di Franco, Giordano Scarciotti and Alessandro Astolfi
}

\begin{abstract}
In this paper the problem of controlling differential-algebraic systems with index-1 is addressed. The proposed technique is based on the interpretation of differentialalgebraic systems as the feedback interconnection of a differential system and an algebraic system. In this framework, the algebraic variable can be treated as an external disturbance acting on the differential system. A direct consequence of this approach is that the control problem reduces to a classical disturbance attenuation problem with internal stability. We also show that the application of the proposed theory to the linear case yields classical results. Finally, an example inspired by an air suspension system in a truck illustrates the technique.
\end{abstract}

\section{INTRODUCTION}

Differential-algebraic (DA) systems (also known as descriptor systems or singular systems) are composite systems of ordinary differential equations and algebraic equations. Such systems are used to model a multitude of engineering processes, such as mechanical systems [1], chemical processes [2] and electrical networks [3], in which algebraic equations arise from environmental constraints, mass conservation equations or thermodynamic relations.

Although the modelling process is simpler when using differential-algebraic equations (DAEs), numerous complications arise in their numerical simulation and control. An important concept which provides a measure of these difficulties is the concept of index. Loosely speaking, the index indicates the number of differentiations required to reduce a DA system to a system of ordinary differential equations (ODEs), see [4] for a precise definition. In many cases, coordinates reduction techniques reduce the DA system to a minimum set of ordinary differential equations, which can then be used as the basis for simulation and control design. For high index systems, several studies in numerical analysis of DAEs have showed that a special numerical treatment is required to eliminate the constraint "drift" phenomenon, see [5], [6] and [7], and the finite escape time phenomenon, see the recent works [8] and [9]. Futhermore, many existing control results for DA systems rely on the derivation of appropriate state-space realizations of the constrained system. For example in [10] a state space realization has been derived for nonlinear constrained mechanical systems via multiple differentiation of the algebraic equations, while in [11] an

P. Di Franco, G. Scarciotti and A. Astolfi are with the Dept. of Electrical and Electronic Engineering, Imperial College London, London, SW7 2AZ, UK, [pierluigi.di-franco13][g.scarciotti][a.astolfi]@ic.ac.uk.

A. Astolfi is also with DICII, University of Rome "Tor Vergata", Via del Politecnico 1, 00133 Rome, Italy.

This work has been partially supported by the European Union's Horizon 2020 Research and Innovation Programme under grant agreement No 739551 (KIOS CoE). output feedback control methodology has been developed for a general class of DA systems which arise in chemical processes. However, there is a broad class of processes in which a reduction to ODEs may be prevented by model uncertainties or by nonlinearities, hence a direct approach in the DA formulation is needed for the controller design.

Recently, a novel approach has been presented in [12], in which index-1 DA systems have been interpreted as the feedback interconnection of a differential system and an algebraic system. The notion of $\mathcal{L}_{2}$-gain for the algebraic system allows the direct application of the small gain theorem for stability analysis. Based on this idea, in this paper the algebraic variable is treated as an external disturbance. As a consequence the control problem of DA systems reduces to a classical disturbance attenuation problem with internal stability for differential systems, the solution of which is given by means of an Hamilton-Jacobi-Isaacs inequality in the most general case. The disturbance attenuation problem has been studied by many authors for differential systems, see [13], [14] and [15] for input affine nonlinear systems, while $\mathcal{H}_{\infty}$ control for a general class of nonlinear systems has been treated in [16], [17] and [18]. All these results may be applied to the framework considered in this paper.

For completeness, note that numerous authors have studied the $\mathcal{H}_{\infty}$ control problem for DA systems. In [19], sufficient conditions for the existence of the solution of the $\mathcal{H}_{\infty}$ control problem have been derived for linear DA systems, while [20] obtained similar results using linear matrix inequalities. In [21] the authors have derived necessary and sufficient conditions for the existence of a controller solving the standard nonlinear $\mathcal{H}_{\infty}$ control problem for a general class of nonlinear DA system, considering both state and output feedback, in terms of Hamilton-Jacobi inequalities. More recently, $\mathcal{H}_{\infty}$ control and robust adaptive control for a class of nonlinear DA systems with external disturbances and parametric uncertainties have been studied in [22]. However, we stress that the novelty of this work is the reduction of the control problem of index-1 DA systems to a classical disturbance attenuation problem with internal stability for a differential system. Moreover, in the special case of linear DA systems, classical results can be interpreted as a particular case of the presented framework.

As an example of application we study the control problem for an air suspension system for trucks. Some of the challenges in control of air suspensions are due to nonlinearities and uncertainties in the model equations, which restricts their use to basic operations such as vehicle lifting for passenger entry and exit or to optimize the comfort, 
while other advanced control tasks such as isolating road harshening are provided by different suspension systems. However, according to many authors (see [23], [24], [25] and references therein for more insight on this topic), robust control which solve the aforementioned problems has not been solved in an analytical way.

The outline of the paper is as follows. In Section II we recall some definitions and preliminary results adapted from [12]. In Section III we give the main result in terms of solution of a Hamilton-Jacobi Isaacs inequality. The application of the theory to the linear case leads to classical results. In Section IV the control design for an air suspension system is presented and simulation results are shown. Finally, in Section V we report our conclusions.

Notation. We use standard notation. The superscript $T$ represents the transposition operator. The symbols $\mathbb{R}_{>0}$ and $\mathbb{R}_{\geq 0}$ indicate, respectively, the set of strictly positive real numbers and the set of non-negative real numbers. Given a function $f: \mathbb{R}^{n} \rightarrow \mathbb{R}$ and a manifold $\mathcal{M}$, the symbol $\left.f\right|_{\mathcal{M}}$ indicates the restriction of $f$ to $\mathcal{M}$. Given a matrix $A$ the symbols $\underline{\sigma}(A)$ and $\bar{\sigma}(A)$ represent the smallest and largest singular value, respectively, of the matrix $A$.

\section{PRELIMINARIES}

In this section we introduce some preliminaries which are instrumental for the remaining of the paper. We first recall some useful definitions regarding the concept of $\mathcal{L}_{2^{-}}$ gain of a nonlinear differential system. We formulate the control problem for a general class of index-1 DA systems and we then recall some results on the stability analysis of DA systems based on [12].

\section{A. Some definitions}

We recall here the definition of $\mathcal{L}_{2}$-norm.

Definition 1. (Definition 1.1 .1 in [18]) The set $\mathcal{L}_{2}[0, \infty$ ) consists of all functions $f: \mathbb{R}_{\geq 0} \rightarrow \mathbb{R}$ which are measurable and satisfy

$$
\int_{0}^{\infty}|f(t)|^{2} d t<\infty
$$

We now give the definition of $\mathcal{L}_{2}$-gain in the spirit of Lemma 3.2.4 of [18].

Definition 2. Let $V$ be a smooth storage function that is nonnegative and with a strict minimizer at $x=0$. The nonlinear system

$$
\begin{aligned}
& \dot{x}=f(x, u), \\
& y=h(x),
\end{aligned}
$$

with state $x(t) \in \mathbb{R}^{n}$, input $u(t) \in \mathbb{R}^{p}$ and output $y(t) \in \mathbb{R}^{m}$ has $\mathcal{L}_{2}$-gain less than $\gamma$ if it is locally dissipative with respect to the supply rate $s(u, h(x))=\gamma^{2}\|u\|^{2}-\|h(x)\|^{2}$, i.e. the inequality

$$
V_{x} f(x, u) \leq s(u, h(x))
$$

holds for all $(x, u)$ in a neighborhood of the origin.

\section{B. Problem formulation}

Consider an index-1 differential-algebraic system in semiexplicit form described by the equations

$$
\begin{aligned}
\dot{x} & =f(x, w, u), \\
0 & =h(x, w, u),
\end{aligned}
$$

where $x(t) \in \mathbb{R}^{n}$ is the differential variable, $w(t) \in \mathbb{R}^{m}$ is the algebraic variable and $u(t) \in \mathbb{R}^{p}$ is the vector of control inputs. Here the mappings $h: \mathbb{R}^{n} \times \mathbb{R}^{m} \times \mathbb{R}^{p} \rightarrow \mathbb{R}^{m}$ and $f: \mathbb{R}^{n} \times \mathbb{R}^{m} \times \mathbb{R}^{p} \rightarrow \mathbb{R}^{n}$ are assumed to be smooth. In addition, we also assume that the origin is the only equilibrium point and that

$$
\operatorname{rank}\left(\frac{\partial h(x, w, 0)}{\partial w}\right)=m,
$$

for all $x(t) \in \mathbb{R}^{n}$ and $w(t) \in \mathbb{R}^{m}$, which means that the system $h(x, w, 0)=0$ does not contain redundant equations. Note that, under these assumptions, the DA system (3) can be rewritten as

$$
\begin{aligned}
\dot{x} & =f(x, w, u), \\
0 & =h_{0}(x, u)+\hat{h}(x, w, u) w,
\end{aligned}
$$

where $h_{0}(x, u)=h(x, 0, u)$ and $\hat{h}: \mathbb{R}^{n} \times \mathbb{R}^{m} \times \mathbb{R}^{p} \rightarrow \mathbb{R}^{m \times m}$ is a smooth mapping such that $\hat{h}(x, w, 0)$ is full rank. The control action in (5) is to be provided by a static feedback controller

$$
u=\Phi(x, w),
$$

such that the closed-loop system (5)-(6) is locally asymptotically stable at the equilibrium. This problem is referred to as the feedback control problem.

\section{Stability analysis}

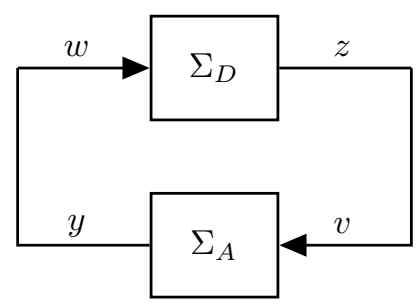

Fig. 1: Feedback interconnection representation of the DA system (5) [12].

We recall here some results of [12] with some minor differences due to the presence of the control action. The DA system (5) can be schematically represented as in Figure 1, where

$\Sigma_{D}:\left\{\begin{aligned} \dot{x} & =F(x, w, u)= \\ & =f(x, w, u)+\psi(x, w)\left(h_{0}(x, u)+\hat{h}(x, w, u) w\right), \\ z & =h_{0}(x, u),\end{aligned}\right.$

has control input $u(t) \in \mathbb{R}^{p}$, disturbance input $w(t) \in \mathbb{R}^{m}$ 
and output $z(t) \in \mathbb{R}^{m}$, and

$$
\Sigma_{A}:\left\{\begin{array}{l}
0=v+\hat{h}(x, w, u) w, \\
y=w
\end{array}\right.
$$

has input $v=z$ and output $y=w$ and it is "parametrized" by $u$ and $x$. Note that, according to [12], the mapping $\psi$ : $\mathbb{R}^{n} \times \mathbb{R}^{m} \rightarrow \mathbb{R}^{n \times m}$ affects the $\mathcal{L}_{2}$-gain of subsystem (7) but it has no effect on the DA interconnection. We recall here a preliminary result.

Lemma 1. (Adapted from Lemma 1 in [12]) Consider the system (6)-(8). Suppose the control input $u$ in equation (6) is such that

$$
\min _{(x, w) \in \mathbb{R}^{n+m}} \underline{\sigma}(\hat{h}(x, w, u)) \geq \epsilon,
$$

for some $\epsilon>0$. Let $\gamma_{A}$ be the $\mathcal{L}_{2}$-gain of system $\Sigma_{A}$ from the input $v$ to the output $w$. Then $\gamma_{A}$ is such that

$$
\gamma_{A} \leq \frac{1}{\epsilon}<+\infty
$$

In what follows we assume that system (7) has the following detectability property.

Assumption 1. Any bounded trajectory $x(t)$ of the system

$$
\dot{x}=F(x(t), 0, u(t))
$$

satisfying

$$
h_{0}(x(t), u(t))=0
$$

for all $t \geq 0$, is such that

$$
\lim _{t \rightarrow \infty} x(t)=0 .
$$

A useful result on the stability analysis of DA systems is the following.

Theorem 1. (Adapted from Theorem 3 in [12]) Consider the system (5) and assume that condition (9) holds for some $\epsilon>0$. Suppose that Assumption 1 holds in a closed and bounded set $\Omega$ and that the $\mathcal{L}_{2}$-gain $\gamma_{D}$ of the system described by equation (7) satisfies the inequality

$$
\gamma_{D}<\epsilon,
$$

for some function $\psi(x, w)$. Then the origin is a locally asymptotically stable equilibrium point.

\section{MAIN RESULT}

In this section we address the problem of finding a controller which renders the closed-loop system (3)-(6) locally asymptotically stable at the origin.

According to the feedback interpretation given in Section II, the algebraic variable can be seen as a disturbance for system (7). The following theorem shows that the control problem of the DA system (5) is reduced to a classical disturbance attenuation problem with internal stability of the ordinary differential system (7).

Theorem 2. Consider the closed-loop DA system (5)-(6). Assume that Assumption 1 holds. Let $V: \mathbb{R}^{n} \rightarrow \mathbb{R}$ be a smooth positive definite function, defined in a neighborhood of the origin and such that $V(0)=0$ and $V_{x}(0)=0$. Let now $H: \mathbb{R}^{n} \times \mathbb{R}^{n} \times \mathbb{R}^{m} \times \mathbb{R}^{p} \rightarrow \mathbb{R}$ be the Hamiltonian function associated with system $\Sigma_{D}$ and defined as

$H\left(x, V_{x}^{\top}, w, u\right)=V_{x} F(x, w, u)+\left\|h_{0}(x, u)\right\|^{2}-\gamma_{D}^{2}\|w\|^{2}$.

If the control action (6) is such that

1) inequality (9) holds for some $\epsilon>0$,

2) $H\left(x, V_{x}^{\top}, w, \Phi(x, w)\right) \leq 0$ for $\gamma_{D}<\epsilon$ and for some $\psi(x, w)$,

then the trajectory of the closed-loop DA system (5)-(6) is locally asymptotically stable at the equilibrium.

Theorem 2 shows that to solve the control problem of an index-1 DA system in form (5), under some appropriate detectability properties, the control action may be provided in such a way that the effect of the exogenous signal $w$ on the $\mathcal{L}_{2}$-gain of the differential subsystem is sufficiently small. This problem is known as the disturbance attenuation problem with internal stability. Numerous results have been provided in the literature for such a problem, see for example [13], [16] and references therein.

\section{A. Linear case}

In the case of linear DA systems, the application of Theorem 2 yields classical results which can be reinterpreted in this framework. Consider an index-1 linear DA system of the form

$$
\begin{aligned}
& \dot{x}=A x+B u+L w, \\
& 0=C x+D u+R w,
\end{aligned}
$$

with $A \in \mathbb{R}^{n \times n}, B \in \mathbb{R}^{n \times p}, L \in \mathbb{R}^{n \times m}, C \in \mathbb{R}^{m \times n}$, $D \in \mathbb{R}^{m \times p}$ and $R \in \mathbb{R}^{m \times m}$, and $R$ full rank by (4). We aim to find a linear feedback control law

$$
u=K x,
$$

such that the closed loop system (13)-(14) is asymptotically stable. For such a system the following result holds.

Corollary 1. If the pair

$$
\left(A-L R^{-1} C, B-L R^{-1} D\right),
$$

is stabilizable then there exists some matrix $K$ such that the closed loop system (13)-(14) is asymptotically stable .

Remark 1. Condition (15) is also necessary. In fact, by manipulating the algebraic equation in (13) yields

$$
w=-R^{-1}(C x+D u),
$$

which replaced in the differential equation of (13) yields

$$
\dot{x}=\left(A-L R^{-1} C\right) x+\left(B-L R^{-1} D\right) u,
$$

which is asymptotically stabilizzable if and only if the pair $\left(A-L R^{-1} C, B-L R^{-1} D\right)$ is stabilizzable.

\section{EXAMPLE}

Consider the air suspension with interconnected components shown in Figure 2. Its basic operation is such that 
the pressure inside the air spring is adjusted to balance the load weight by actuating valves which let the air flow from an external accumulator into the air bellow and from the air bellow to the external environment. The nonlinear equations of motion governing the dynamic behaviour of the air suspension system in Figure 2 are taken from [26] and are given by the equations

$$
\begin{aligned}
M \ddot{\xi} & =-M g_{v}-\mu \dot{\xi}+F_{e}(p, \xi), \\
\dot{m}_{a i r} & =\frac{P_{s}}{\sqrt{R_{a} T}} \Psi\left(\frac{p}{P_{s}}\right) u_{1}-\frac{p}{\sqrt{R_{a} T}} \Psi\left(\frac{P_{a t m}}{p}\right) u_{2}, \\
p V(p, \xi) & =m_{a i r} R_{a} T
\end{aligned}
$$

where $\xi(t) \in \mathbb{R}$ is the height of the suspension, $M$ the mass load, $\mu$ a viscous friction coefficient, $p(t) \in \mathbb{R}$ the air spring pressure, $P_{s}$ and $P_{a t m}$ the supply and atmospheric pressures respectively, $m_{\text {air }}(t) \in \mathbb{R}$ the mass of air inside the spring, $R_{a}$ the air specific gas constant, $T$ the temperature and $g_{v}$ the gravitational acceleration. The air is modelled as a perfect gas which flows in and out the air suspension through a Venturi, at constant temperature. The signals $u_{1}(t) \in \mathbb{R}$ and $u_{2}(t) \in \mathbb{R}$ control the amount of air flowing through the valves. The mapping $\Psi(\cdot)$, which models the nonlinearity of the solenoid valves, is

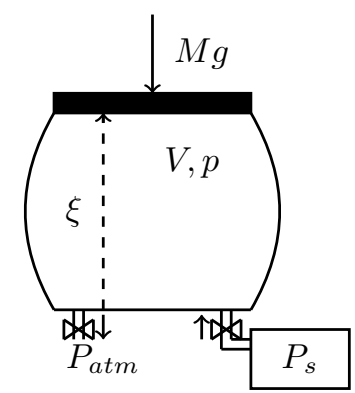

Fig. 2: Sketch of the air suspension system.

$$
\Psi\left(\frac{p_{u}}{p_{d}}\right)=\sqrt{\frac{2 \gamma}{\gamma-1}\left(\left(\frac{p_{u}}{p_{d}}\right)^{\frac{2}{\gamma}}-\left(\frac{p_{u}}{p_{d}}\right)^{\frac{\gamma+1}{\gamma}}\right)}
$$

where $\gamma$ is the specific heat ratio of the air, and $p_{u}$ and $p_{d}$ are the upstream and downstream pressures, respectively. The mapping

$$
F_{e}(p, \xi)=p A_{e}(p, \xi)
$$

represents the force developed by the air spring, where $A_{e}(p, \xi)$ is the effective area which, in general, is a nonlinear function of the pressure $p$ and the height $\xi$ and its value can be calculated from the load charts provided by the manufacturer. Observe that there is no dynamic equation of the pressure $p$ and it is not trivial to reduce the DA equations (17) to ordinary differential equations as the volume $V(p, \xi)$ depends itself on the pressure and on the height. In addition, the mapping $V(p, \xi)$ is usually not explicitly available but is provided in charts by the manufacturer. However, due to the accumulator, pipes and cushioning chambers, a minimum volume $V_{0}$ is supposed to exist.

Consider system (17) and assume the variables $\xi, \dot{\xi}, m_{\text {air }}$ and $p$ are measurable. We aim to find a feedback control law

$$
u=\left[\begin{array}{l}
u_{1} \\
u_{2}
\end{array}\right]=\Phi\left(\xi, \dot{\xi}, m_{a i r}, p\right),
$$

such that

$$
\begin{aligned}
& \lim _{t \rightarrow \infty} m_{\text {air }}(t)-m_{\text {air }}^{*}(t)=0, \\
& \lim _{t \rightarrow \infty} \dot{\xi}(t)=0, \\
& \lim _{t \rightarrow \infty} p(t)-p^{*}(t)=0,
\end{aligned}
$$

where $m_{\text {air }}^{*}(t)$ is a given reference signal and $p^{*}(t)$ is the pressure equilibrium setpoint. To this end, let

$$
\left[\xi, \dot{\xi}, m_{a i r}, p, m_{\text {air }}^{*}\right]^{\top}=\left[x_{1}, x_{2}, x_{3}, w, x_{3}^{*}\right]^{\top},
$$

and

$$
g(w)=\left[\begin{array}{c}
\frac{P_{s}}{\sqrt{R_{a} T}} \Psi\left(\frac{w}{P_{s}}\right) \\
-\frac{w}{\sqrt{R_{a} T}} \Psi\left(\frac{P_{a t m}}{w}\right)
\end{array}\right] .
$$

Then system (17) can be rewritten in state space form as the nonlinear DA system

$$
\begin{aligned}
\dot{x}_{1} & =x_{2}, \\
\dot{x}_{2} & =-g_{v}-\frac{\mu}{M} x_{2}+\frac{A_{e}\left(w, x_{1}\right)}{M} w, \\
\dot{x}_{3} & =g(w) u, \\
0 & =R_{a} T x_{3}-V\left(w, x_{1}\right) w .
\end{aligned}
$$

On the basis of Theorem 2, the feedback controller

$$
\Phi\left(x_{3}^{*}, x_{3}, w\right)=g(w)\left(g(w)^{\top} g(w)\right)^{-1}\left(\dot{x}_{3}^{*}-k\left(x_{3}-x_{3}^{*}\right)\right),
$$

with $k>0$ is such that conditions (19) hold for all $x_{1}(0) \in \mathbb{R}$, $x_{2}(0) \in \mathbb{R}, x_{3}(0) \in \mathbb{R}$ and $p(0) \in \mathbb{R}$.

Simulations have been conducted to verify the effectiveness of the proposed control algorithm. The following set of parameters have been used:

$$
\left[\begin{array}{c}
P_{s} \\
P_{a t m} \\
\mu \\
\gamma \\
T \\
R_{a} \\
V_{0} \\
k
\end{array}\right]=\left[\begin{array}{c}
5 \cdot 10^{5} \mathrm{~Pa} \\
10^{5} \mathrm{~Pa} \\
100 \\
1.41 \\
298.15 \mathrm{~K} \\
287.058 \frac{\mathrm{J}}{\mathrm{kgK}} \\
0.001 \mathrm{~m}^{3} \\
100
\end{array}\right]
$$

with initial conditions

$$
\left[\begin{array}{c}
\xi(0) \\
\dot{\xi}(0) \\
m_{\text {air }}(0) \\
p(0)
\end{array}\right]=\left[\begin{array}{c}
0.3 \mathrm{~m} \\
0 \frac{\mathrm{m}}{\mathrm{s}} \\
3.675 \cdot 10^{-3} \mathrm{~kg} \\
10^{5} \mathrm{~Pa}
\end{array}\right]
$$

The control objective is to track a step reference signal $m_{\text {air }}^{*}(t)$ in the presence of a constant payload $M=150 \mathrm{~kg}$. Simulation results are shown in Figures 3, 4, 5, 6, 7 and 8. The reference $m_{\text {air }}^{*}$ and the mass of air $m_{\text {air }}$ are given in Figure 3 with 
dashed line and solid line, respectively. In Figures 4 and 5 the zooming views of the first and second steps are also given. These reveal a fast rate of convergence due to the high gain $k=100$. The time histories of $p$ and $\dot{\xi}$, shown in Figures 6 and 7 , illustrate the convergence to the equilibrium. Note that the rate of convergence is slower than that of $m_{\text {air }}$. Finally, Figure 8 shows the time history of the height $\xi$.

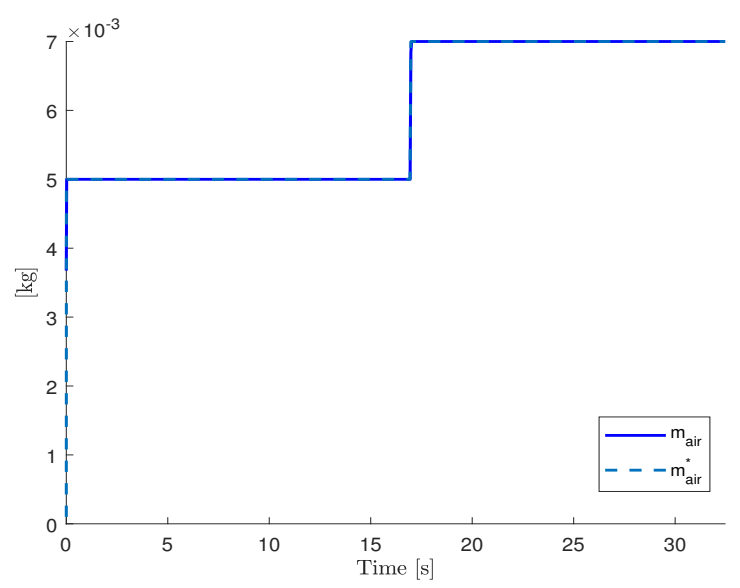

Fig. 3: Time history of $m_{a i r}(t)$ and its reference $m_{\text {air }}^{*}(t)$.

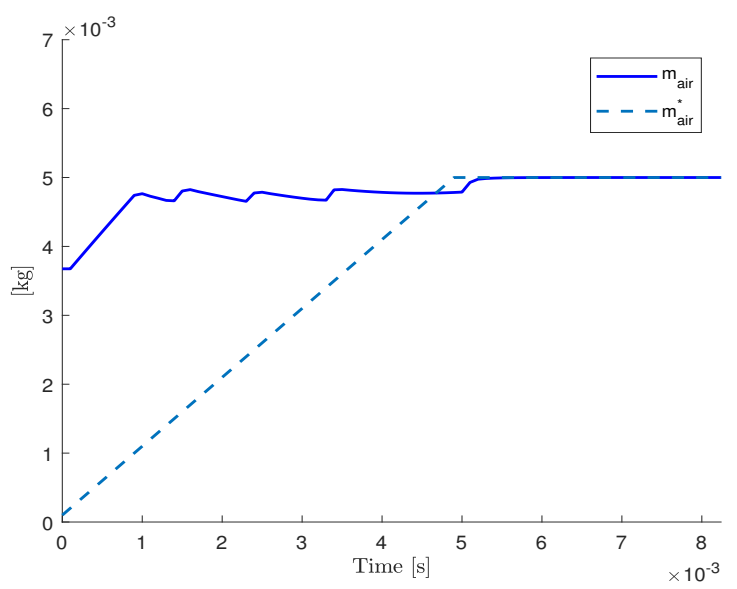

Fig. 4: Time history of $m_{\text {air }}(t)$ and its reference $m_{\text {air }}^{*}(t)$ : first zoom.

\section{CONCLUSIONS}

By interpreting a DA system as the feedback interconnection of a differential system and an algebraic system, the problem of control of nonlinear DA systems with index-1 has been reduced to the classical disturbance attenuation problem with internal stability, for which conditions have been derived using the Hamilton-Jacobi-Isaacs inequality. We have also shown that classical results for linear DA systems can be reinterpreted in this framework. Finally, we have provided an example of application inspired by an air suspension system for a truck and simulation results have been discussed.

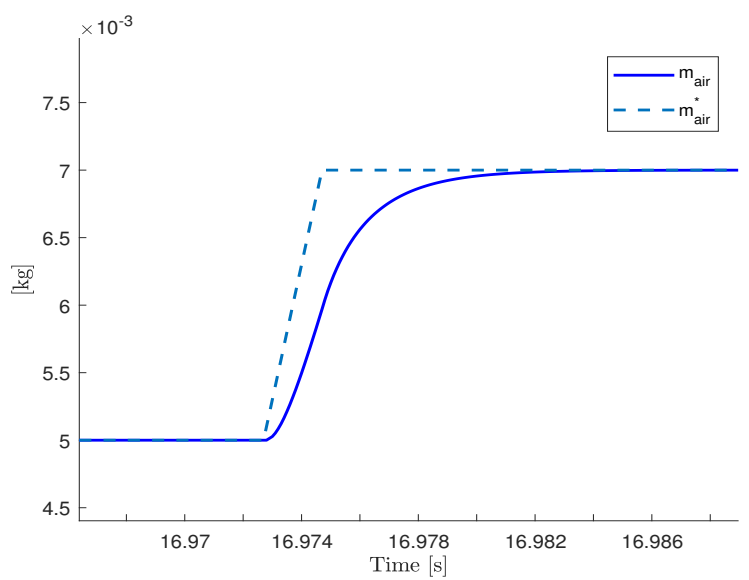

Fig. 5: Time history of $m_{a i r}(t)$ and its reference $m_{a i r}^{*}(t)$ : second zoom.

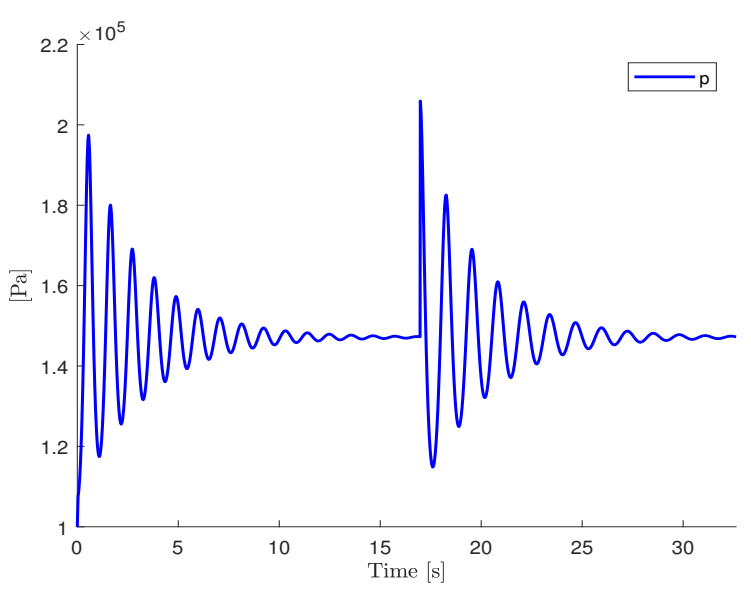

Fig. 6: Time history of $p(t)$.

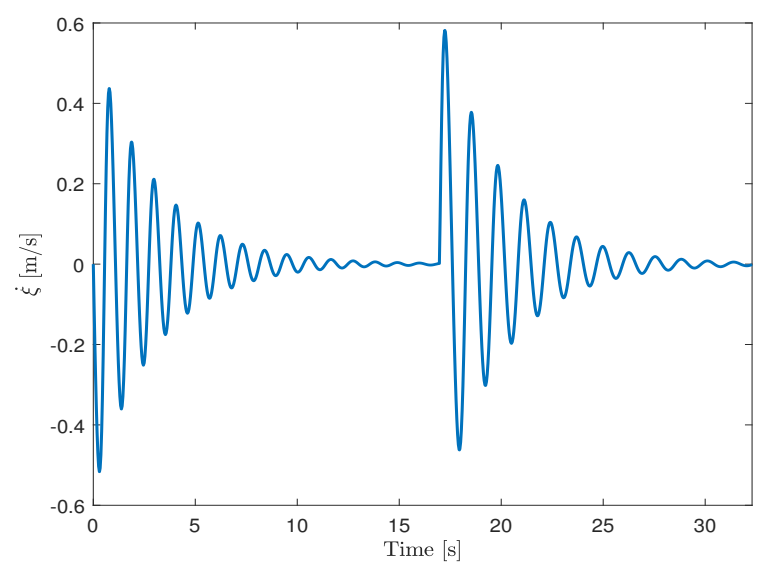

Fig. 7: Time history of $\dot{\xi}(t)$.

\section{REFERENCES}

[1] W. Blajer, "Index of differential-algebraic equations governing the dynamics of constrained mechanical systems," Applied Mathematical 


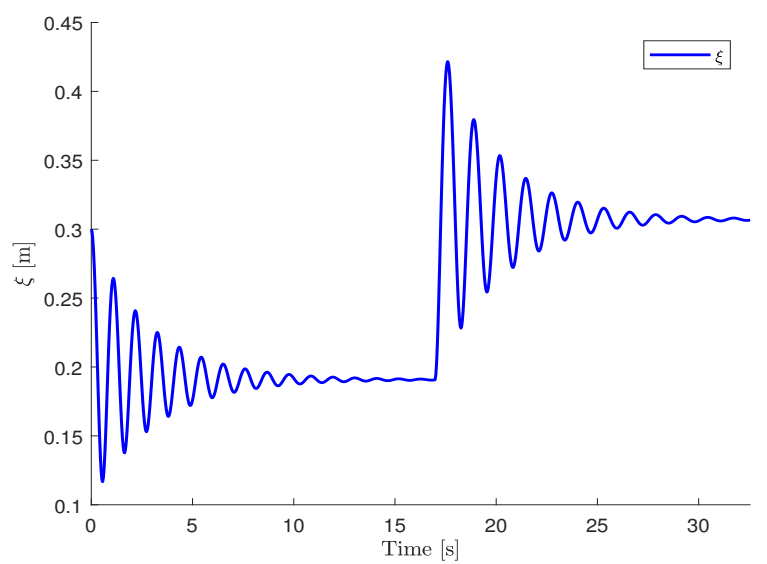

Fig. 8: Time history of $\xi(t)$.
IEEE Conference on Decision and Control, vol. 3, Dec 1994, pp. 2251-2256.

[20] I. Masubuchi, Y. Kamitane, A. Ohara, and N. Suda, " $\mathcal{H}_{\infty}$ control for descriptor systems: A matrix inequalities approach," Automatica, vol. 33, no. 4, pp. 669-673, Apr. 1997.

[21] H.-S. Wang, C.-F. Yung, and F.-R. Chang, $\mathcal{H}_{\infty}$ Control for Nonlinear Descriptor Systems (Lecture Notes in Control and Information Sciences). Secaucus, NJ, USA: Springer-Verlag New York, Inc., 2006.

[22] L. Sun and Y. Wang, "An undecomposed approach to control design for a class of nonlinear descriptor systems," International Journal of Robust and Nonlinear Control, vol. 23, no. 6, pp. 695-708, 2013.

[23] H. Kim and H. Lee, "Height and leveling control of automotive air suspension system using sliding mode approach," IEEE Transactions on Vehicular Technology, vol. 60, no. 5, pp. 2027-2041, Jun 2011.

[24] H. Kim, H. Lee, and H. Kim, "Asynchronous and synchronous load leveling compensation algorithm in airspring suspension," in 2007 International Conference on Control, Automation and Systems, Oct 2007, pp. 367-372.

[25] H. Porumamilla and A. G. Kelkar, "Robust control and $\mu$ analysis of active pneumatic suspension," in Proceedings of the 2005, American Control Conference, 2005., June 2005, pp. 2200-2205 vol. 3.

[26] J. Aslund and E. Frisk, "An observer for non-linear differential-algebraic systems," Automatica, vol. 42, no. 6, pp. 959 - 965, 2006.

Modelling, vol. 16, no. 10, pp. 70-77, 1992.

[2] A. Kumar and P. Daoutidis, Control of Nonlinear Differential Algebraic Equation Systems : An Overview. Dordrecht: Springer Netherlands, 1998, pp. 311-344.

[3] R. Riaza, Differential-Algebraic Systems: Analytical Aspects and Circuit Applications. River Edge, NJ, USA: World Scientific Publishing Co Pte ltd, 2008.

[4] V. Mehrmann, Index Concepts for Differential-Algebraic Equations. Berlin, Heidelberg: Springer Berlin Heidelberg, 2015, pp. 676-681.

[5] J. Baumgarte, "Stabilization of constraints and integrals of motion in dynamical systems," Computer Methods in Applied Mechanics and Engineering, vol. 1, no. 1, pp. 1 - 16, 1972.

[6] C. W. Gear, Numerical Initial Value Problems in Ordinary Differential Equations. Upper Saddle River, NJ, USA: Prentice Hall PTR, 1971.

[7] K. E. Brenan, S. L., and L. R. Petzold, Numerical solution of initialvalue problems in differential-algebraic equations, ser. Classics in applied mathematics. Philadelphia, Pa.: SIAM, 1996.

[8] P. Di Franco, G. Scarciotti, and A. Astolfi, "A globally stable convergent algorithm for the integration of constrained mechanical systems," in IEEE 2018 American Control Conference.

[9] _ - "Discretization schemes for constraint stabilization in nonlinear differential-algebraic systems," in IEEE 2018 European Control Conference.

[10] N. H. McClamroch, "Feedback stabilization of control systems described by a class of nonlinear differential-algebraic equations," Systems Control Letters, vol. 15, no. 1, pp. 53 - 60, 1990.

[11] A. Kumar and P. Daoutidis, "Feedback control of nonlinear differentialalgebraic equation systems," AIChE Journal, vol. 41, no. 3, pp. 619-636, 1995.

[12] P. Di Franco, G. Scarciotti, and A. Astolfi, "A note on the stability of nonlinear differential-algebraic systems," in 20th IFAC World Congress, Toulouse, France, July 9-14, 2017.

[13] A. Isidori and A. Astolfi, "Disturbance attenuation and $\mathcal{H}_{\infty}$ control via measurement feedback in nonlinear systems," IEEE Transactions on Automatic Control, vol. 37, no. 9, pp. 1283-1293, Sep 1992.

[14] A. Isidori, " $\mathcal{H}_{\infty}$ control via measurement feedback for affine nonlinear systems," vol. 4, pp. 553 - 574, 071994.

[15] A. van der Schaft, "On a state space approach to nonlinear $\mathcal{H}_{\infty}$ control," Systems Control Letters, vol. 16, no. 1, pp. 1 - 8, 1991.

[16] A. Isidori and W. Kang, " $\mathcal{H}_{\infty}$ control via measurement feedback for general nonlinear systems," IEEE Transactions on Automatic Control, vol. 40, no. 3, pp. 466-472, Mar 1995.

[17] J. A. Ball, J. W. Helton, and M. L. Walker, " $\mathcal{H}_{\infty}$ control for nonlinear systems with output feedback," IEEE Transactions on Automatic Control, vol. 38, no. 4, pp. 546-559, Apr 1993.

[18] A. J. van der Schaft, $\mathcal{L}_{2}$-Gain and Passivity Techniques in Nonlinear Control, 1st ed. Secaucus, NJ, USA: Springer-Verlag New York, Inc., 1996.

[19] K. Takaba, N. Morihira, and T. Katayama, " $\mathcal{H}_{\infty}$ control for descriptor systems: A J-spectral factorization approach," in Proceedings of 33rd 